\title{
Parental Age and the Risk of Autism Spectrum Disorder in Oman A case-control study
}

Watfa Al-Mamari, ${ }^{1}$ *Ahmed B. Idris, ${ }^{1}$ Aala' A. Al-Zadjali, ${ }^{3}$ Saquib Jalees, ${ }^{1}$ Sathiya Murthi, ${ }^{4}$ Muna Al-Jabri, ${ }^{2}$ Ahlam Gabr, ${ }^{1}$ Eric Fombonne ${ }^{5}$

\begin{abstract}
Objectives: This study aimed at evaluating advanced parental age as a risk factor for autism spectrum disorder (ASD) in an Omani cohort. Methods: This case-control study compared 278 ASD cases with 722 gendermatched controls, retrieved from the electronic records of the Developmental Paediatric Clinic, Sultan Qaboos University Hospital, Muscat, Oman, between January 2015 and June 2016. Results: Most ASD cases were male (76.6\%) and mostly diagnosed between 3-4 years of age, with more than $50 \%$ of the cases originating from Muscat and Batinah governorates. Compared to controls, mothers from the case group had significantly higher educational levels (post-secondary education versus high school/no formal education: odds ratio $[\mathrm{OR}]=1.62,95 \%$ confidence interval [CI]: 1.197-2.192). In a multivariate logistic regression, the OR of maternal age as a risk for ASD increased dramatically with advancing age category (using age $<25$ as reference, $\mathrm{OR}=3.39,6.12,7.86$ and 13.13 for age categories 25-29, 30-34, 35-39 and $\geq 40$ years, respectively). The ORs of advancing paternal age as a risk for ASD were also statistically significant (using age $<30$ as reference, $O R=2.20,2.36$ and 3.12 for age categories 30-34, $35-39$ and 40-44 years, respectively); however, there was a drop in the effect with paternal age $\geq 45$ years (OR $=1.42$; 95\% CI: 0.64-3.15). Conclusion: Both maternal and paternal increased age were associated with a higher risk of ASD; however, the association was more pronounced and more consistent with advanced maternal age compared to paternal age.
\end{abstract}

Keywords: Autism; Parental Age; Case-Control Study; Oman.

\section{AdvanCeS IN KNOWLEdGE}

This study tested the association between advancing parental age and autism spectrum disorder (ASD) in an Omani population characterised by a younger marriage and conception age and a high proportion of consanguineous unions.

Advanced maternal age was associated with a significantly increased risk of having a child with autism in a dose-response manner.

A significant paternal age effect was also found but it was smaller than that for maternal age and was not significant anymore at age $\geq 45$ years, a finding that warrants further examination.

\section{Application to Patient Care}

Consideration of the relationship between advanced parental age and risk of having a child with ASD can assist in counselling with respect to pregnancy at an older age.

A UTISM SPECTRUM DISORDER (ASD) IS A neurodevelopmental disorder with hallmark features of deficits in social interaction and social communication along with restricted, repetitive patterns of behaviour, interests or activities. ${ }^{1}$ Globally, the prevalence of ASD was estimated to be 62 per 10,000 while it was 185 per 10,000 (one in 54) in the United States., ${ }^{2,3}$ In Oman, the prevalence of ASD, based on 2019 estimates, was 20.35 per 10,000 children and was 15-fold greater than estimates in 2011. ${ }^{4}$ The increment in ASD prevalence worldwide is attributed mainly to an increase of diagnostic facilities and services, awareness and diagnostic substitution. ${ }^{5}$

Many perinatal factors were found to be associated with ASD but none has been proven to be the causative aetiologic factor. For instance, maternal obesity, maternal gestational diabetes, caesarean section, preterm deliveries, congenital malformations, low birth weight and low APGAR scores were observed to be associated with an increased risk of ASD in children..$^{6-9}$ Interestingly, whether those factors have a causal effect or play a secondary role in shaping the phenotype in a genetically susceptible individual is still not established. Hence, it was suggested to combine the factors that are implicated in the ASD risk in one model to examine their effects jointly. ${ }^{10}$

The association between advanced parental age and ASD has been examined extensively. Initial reports were highlighted by Reichenberg et al. with observations further tested in different Western populations. ${ }^{11-14}$ A meta-analysis was conducted, utilising a dose-response analysis to investigate the 
effect of increasing parental age on the risk of autism; the results showed that an increase of 10 years in maternal age was associated with an 18\% higher risk of autism. ${ }^{15}$ The effect was more obvious in fathers, where an increase of 10 years was associated with $21 \%$ higher risk of autism. ${ }^{15}$ To overcome the effect of the other parent's age as a confounding factor, Sandin et al. examined the joint effect of maternal and paternal age in a population-based study across five countries. ${ }^{14}$ The results revealed that for mothers older than 40 years, the relative risk (RR) of autism in offspring increased in a U-shaped pattern with younger and older fathers. This was contrasted by effect among fathers $\geq 45$ years, where the RR increases monotonically with increasing maternal age. ${ }^{14}$

Despite several publications documenting the association between advanced parental age and ASD, the underpinning mechanism is still ill-defined. ${ }^{15}$ Copyerrors in the germ cell resulting in de novo mutations in the offspring of older males was proposed as one possible underpinning mechanism. ${ }^{16,17}$ However, the effects of replication errors in the germline alone can not explain the full epidemiological data; rather, it is portrayed to be multifactorial. Aside from replication errors, lifetime environmental exposures and epigenetic alterations have been described as explanatory pathways that contribute to the causation of complex disorders such as autism. ${ }^{18}$

Most studies about the effect of advanced parental age in ASD risk were conducted on Western samples with limited data from Middle Eastern populations that possess interesting differences in demographic features. In Oman, for instance, the population median age is 26.3 years and considered as a young population compared to those of Denmark, USA, Sweden and Norway, where the population median age is 41.1, 37.4, 41 and 39 years, respectively. ${ }^{19}$ The current study aimed to test the association between advancing parental age and ASD risk in an Omani cohort that represents one Middle Eastern population. Specific characteristics of this Omani population's demographic features, such as younger age and younger marriage and conception ages, combined with high consanguinity rates, provided a unique opportunity to replicate parental age findings in autism in a different population and to identify moderating factors that might mitigate the risk. $^{20}$

\section{Methods}

This case-control study included 278 cases with ASD and 722 non-autistic gender-matched controls. All Omani children aged less than 14 years and diagnosed at the Developmental Paediatric Clinic, Sultan Qaboos
University Hospital, Muscat, Oman, were eligible to be included in the study as cases. Data were obtained from electronic records between January 2015 and June 2016.

ASD diagnosis was confirmed by a multidisciplinary team assessment to ensure fulfilment of Diagnostic and Statistical Manual of Mental Disorders-5 diagnostic criteria. ${ }^{1}$ The diagnostic teams were composed of a psychologist, speech and language therapist, occupational therapist, a paediatrician experienced in autism and a certified developmental paediatrician. The diagnostic procedure included the utilisation of Autism Diagnostic Interview-Revised $\left(\mathrm{ADI}^{\mathrm{TM}}-\mathrm{R}\right)$ and the Autism Diagnostic Observation Schedule, Second Edition $\left(\mathrm{ADOS}^{\mathrm{TM}}-2\right)$ as the gold standard methods of ASD diagnosis. All simplex cases were included and for multiplex families, one child only among the affected siblings was retained in order to maintain the independence of observations.

The control group was selected from the national registry of the Ministry of Health, Oman, with data derived from the child's electronic health record. Oman has a free universal healthcare system with almost $96 \%$ of deliveries recorded in health facilities. Children's health records contain information completed by health personnel with data stored in two formats: a paper card belonging to the mother and electronic records in the health system. The electronic record is linked to the individual's unique national identifier and contains sociodemographic information along with health information about the mother and infant. ${ }^{21}$ The electronic records of the control group were reviewed to ensure the absence of neurodevelopmental or behavioural disturbances that could be related to or confused with autism. Controls were randomly selected and matched with cases with respect to age, gender and geographical area. Whenever the matching procedure permitted, up to three controls were selected for each case in order to increase statistical power. In some cases, especially at older ages, once controls had been identified from the same geographical area and gender, there were fewer than three available controls, which explains why the average age of controls is slightly lower than that of the cases.

Medical and electronic records data were extracted on a survey sheet. Data included the child's gender, date of birth, date of diagnosis, residence, parents' level of education and age of the child at the time of ASD diagnosis. Data were transferred into an Excel sheet where the age of the parents at birth of their child with ASD was calculated by subtracting the child's age at time of diagnosis from the parents' age at time of diagnosis. For the control group, the age of 
the parents at birth was provided in the child's health card record. The participant's national identifier was substituted with a research identification number to ensure confidentiality, abiding by ethical guidelines.

Data were analysed using Statistical Package for the Social Sciences (SPSS), Version 25.0 (IBM Corp., Armonk, New York, USA). Bivariate associations were evaluated using a chi-squared test for categorical data and $\mathrm{t}$-tests for continuous variables. Then, a series of bivariate logistic regression models were estimated using case status (ASD = 1 and control $=0$ ) as the dependent variable and predictors (child age, delivery mode, parental age, maternal education status level and governorate of residence) that could be acting as confounding variables.

Bivariate associations were estimated between cases and controls in terms of children's demographics, maternal and parental age groups with unadjusted odds ratios (OR) and 95\% confidence intervals (CI). Only those independent variables that had a weak association $(P<0.15)$ with case status were retained for later inclusion in a multiple logistic regression analysis. Finally, a stepwise multiple logistic regression was conducted with case status as the dependent variable, paternal age and maternal age as risk factors of interest and all potential confounding variables were retained in the previous step. The full multiple logistic regression model was evaluated with the loglikelihood and Wald statistics; a $P$ value of $<0.05$ was considered for retaining a variable in the model as statistically significant. Goodness-of-fit of the final model was estimated with the Hosmer-Lemeshow statistic.

Ethical approval was obtained from the Medical Research \& Ethics Committee of the College of Medicine \& Health Sciences, Sultan Qaboos University, Muscat, Oman, and the Centre of Studies and Research, Research Committee, Ministry of Health, Oman (MREC \#752; Unique Identification Code \#5820, respectively).

\section{Results}

A total of 1000 subjects were studied where 278 (27.8\%) were ASD cases and 722 (72.2\%) were controls. Of all included children, $78.0 \%$ were male; the male to female ratio was 3.28:1 in the ASD group. Over half of the cohort resided in Muscat and Batinah governorates (28.4\% and 27.6\%, respectively) [Table 1].

There was no statistically significant difference observed between cases and controls in terms of gender and mode of delivery $(P>0.05)$; however, statistically significant differences were observed in maternal education $(P=0.001)$, categorical maternal and paternal ages $(P<0.001$ each). Despite this study's attempt to match the ages when selecting cases and controls, the child's age was also significantly different between the two groups and, therefore, the child's age was subsequently adjusted for in following analyses [Table 2].

In a multivariate logistical model, with the exception of child age, all independent variables significantly contributed to the model. Highlyeducated mothers were more likely to have a child with ASD compared to school-educated mothers or those with no formal education (adjusted OR $=1.48$, 95\% CI: 1.07-2.07). The odds of having a child with ASD increased significantly with advancing maternal age in a dose-response fashion with the highest risk estimate being for mothers $\geq 40$ years. There was also an increased risk of ASD with increasing paternal age. The magnitude of the risk increase was less for paternal age compared to maternal age (adjusted OR $=1.42-3.12$ versus 3.39-13.13). Furthermore, the OR for fathers in the oldest age group ( $\geq 45$ years)

Table 1: Characteristics of children with autism spectrum disorder and non-autistic matched controls $(\mathrm{N}=1,000)$

\begin{tabular}{lcc} 
Characteristic & \multicolumn{2}{c}{$\mathbf{n}(\%)$} \\
& ASD $(\mathbf{n}=278)$ & Controls $(\mathbf{n}=722)$ \\
$\begin{array}{l}\text { Mean age in } \\
\text { months } \pm \text { SD }\end{array}$ & $58.1 \pm 22.4$ & $53.2 \pm 12.6$ \\
Age category in years & & \\
$0-2$ & $16(5.8)$ & $32(4.4)$ \\
3 & $77(27.7)$ & $225(31.2)$ \\
4 & $83(29.9)$ & $234(32.4)$ \\
5 & $50(18.0)$ & $144(19.9)$ \\
6 & $21(7.6)$ & $63(8.7)$ \\
$\geq 7$ & $31(11.2)$ & $24(3.3)$ \\
Gender & & $155(21.5)$ \\
Female & $65(23.4)$ & $567(78.5)$ \\
Male & $213(76.6)$ & $191(8.4)$ \\
Governorate of residence & $210(29.1)$ \\
Muscat & $74(26.6)$ & $12(1.7)$ \\
Buraimi & $8(2.9)$ & $19.6)$ \\
Batinah & $77(27.7)$ & \\
Dahira & $10(3.6)$ & $(18.1)$ \\
Dhofar & $24(8.6)$ & \\
Dakhiliya & $51(18.3)$ & \\
Sharqia & & \\
\hline
\end{tabular}

$A S D=$ autism spectrum disorder; $S D=$ standard deviation. 
Table 2: Associations between the studied variables and case-control status using bivariate regression $(\mathrm{N}=1,000)$

\begin{tabular}{|c|c|c|c|c|}
\hline \multirow[t]{2}{*}{ Variable } & \multicolumn{2}{|c|}{ n (\%) } & \multirow[t]{2}{*}{$P$ value } & \multirow{2}{*}{$\begin{array}{l}\text { Unadjusted OR } \\
\quad(95 \% \mathrm{CI})\end{array}$} \\
\hline & Controls $(n=722)$ & Cases $(n=278)$ & & \\
\hline Male gender & $567(78.5)$ & $213(76.6)$ & 0.283 & $0.896(0.644-1.246)$ \\
\hline Caesarian section & $140(19.4)$ & $55(19.8)$ & 0.476 & $1.02(0.724-1.452)$ \\
\hline $\begin{array}{l}\text { Maternal education of bachelor's } \\
\text { degree or higher }\end{array}$ & $171(23.7)$ & $93(33.5)$ & 0.001 & $1.62(1.197-2.192)$ \\
\hline \multicolumn{5}{|l|}{ Maternal age category in years } \\
\hline$<25$ & $141(19.5)$ & $7(2.5)$ & $<0.001$ & Reference \\
\hline $25-29$ & $234 .(32.4)$ & $54(19.4)$ & & $4.65(2.06-10.50)$ \\
\hline $30-34$ & $203(28.1)$ & $105(37.8)$ & & $10.42(4.71-23.06)$ \\
\hline $35-39$ & $112(15.5)$ & $80(28.8)$ & & $14.39(6.39-32.39)$ \\
\hline$\geq 40$ & $32(4.4)$ & $32(11.5)$ & & $20.14(8.16-49.71)$ \\
\hline \multicolumn{5}{|l|}{ Paternal age category in years } \\
\hline$<30$ & 194. (26.9) & $19(6.8)$ & $<0.001$ & Reference \\
\hline $30-34$ & $221(30.6)$ & $76(27.3)$ & & $3.51(2.05-6.02)$ \\
\hline $35-39$ & $166(23.0)$ & $88(31.7)$ & & $5.41(3.16-9.26)$ \\
\hline $40-44$ & $77(10.7)$ & $65(23.4)$ & & $8.62(4.85-15.32)$ \\
\hline$\geq 45$ & $64(8.9)$ & $30(10.8)$ & & $4.79(2.52-9.08)$ \\
\hline \multicolumn{5}{|l|}{ Child's age category in years } \\
\hline $0-2$ & $32(4.4)$ & $16(5.8)$ & $<0.001$ & Reference \\
\hline 3 & $225(31.2)$ & 77 (27.7) & & $0.68(0.36-1.32)$ \\
\hline 4 & $234(32.4)$ & 83 (29.9) & & $0.71(0.37-1.36)$ \\
\hline 5 & $144(19.9)$ & $50(18.0)$ & & $0.69(0.35-1.37)$ \\
\hline 6 & $63(8.7)$ & $21(7.6)$ & & $0.67(0.31-1.45)$ \\
\hline$\geq 7$ & $24(3.3)$ & 31 (11.2) & & $2.58(1.16-5.76)$ \\
\hline
\end{tabular}

$O R=$ odds ratio; $C I=$ confidence interval .

was not significant anymore $(P=0.389)$ although the OR estimate remained above $1(\mathrm{OR}=1.42)$ [Table 3]. The child's age was statiscally significant $(P=0.011)$ but weakly predictive of case status although no statistical difference was found for any age-specific stratum. The model fit with the data was good as indicated by the Hosmer-Lemeshow chi-square goodness-of-fit $\left(X^{2}=\right.$ $3.67,8$ degrees of freedom; $P=0.886$ ).

\section{Discussion}

Males constituted $76.6 \%$ of the studied cases, which is in line with the gender distribution of autism in epidemiological studies. ${ }^{22}$ The geographical distribution of autism cases revealed that approximately half reside in the governorates of Muscat and Batinah, which follows the geographical distribution of the population in Oman. ${ }^{23}$ Interestingly, $33.5 \%$ of mothers with children who have ASD had a bachelor's degree or higher educational level, which was significantly higher than the control group (23.7\%; $P=0.001)$. A similar finding was noticed in a study investigating the birth cohort and administrative ASD records in California, USA, where investigators found that educated parents and high socioeconomic status families had significantly lower age of diagnosis for their children, which could be explained by better access to care. ${ }^{24}$ Thus, it can be hypothesised that Omani mothers with higher education levels are reporting their children with ASD symptomatology earlier than less-educated mothers.

Both increasing paternal and maternal ages were associated with increased risk of ASD; however, the effect was stronger and more consistent among mothers. This study's findings showed that increasing maternal age was associated with increased ASD risk 
Table 3: Final model of the multiple logistic regression analysis

\begin{tabular}{|c|c|c|c|}
\hline Variable & $\begin{array}{c}\text { Beta } \\
\text { coefficient }\end{array}$ & $\begin{array}{c}P \\
\text { value }\end{array}$ & $\begin{array}{l}\text { Adjusted OR } \\
\quad(95 \% \text { CI })\end{array}$ \\
\hline $\begin{array}{l}\text { Maternal } \\
\text { education } \\
\text { of bachelor's } \\
\text { degree or } \\
\text { higher }\end{array}$ & 0.396 & 0.020 & $1.48(1.07-2.07)$ \\
\hline \multicolumn{4}{|c|}{ Maternal age category in years } \\
\hline$<25$ & - & - & Reference \\
\hline $25-29$ & 1.220 & 0.004 & $3.39(1.47-7.79)$ \\
\hline $30-34$ & 1.811 & $<0.001$ & $6.12(2.63-14.24)$ \\
\hline $35-39$ & 2.062 & $<0.001$ & $7.86(3.22-19.19)$ \\
\hline$\geq 40$ & 2.575 & $<0.001$ & $13.13(4.63-37.26)$ \\
\hline \multicolumn{4}{|c|}{ Paternal age category in years } \\
\hline$<30$ & - & - & Reference \\
\hline $30-34$ & 0.788 & 0.007 & $2.20(1.24-3.91)$ \\
\hline $35-39$ & 0.857 & 0.006 & $2.36(1.27-4.36)$ \\
\hline $40-44$ & 1.139 & 0.001 & $3.12(1.58-6.16)$ \\
\hline$\geq 45$ & 0.350 & 0.389 & $1.42(0.64-3.15)$ \\
\hline \multicolumn{4}{|c|}{ Child's age category in years } \\
\hline $0-2$ & - & - & Reference \\
\hline 3 & -0.435 & 0.219 & $0.647(0.32-1.30)$ \\
\hline 4 & -0.316 & 0.370 & $0.729(0.37-1.46)$ \\
\hline 5 & -0.323 & 0.382 & $0.724(0.35-1.50)$ \\
\hline 6 & -0.434 & 0.305 & $0.648(0.28-1.48)$ \\
\hline$\geq 7$ & 0.742 & 0.089 & $2.101(0.89-4.94)$ \\
\hline
\end{tabular}

$O R=$ odds ratio; $C I=$ confidence interval .

in a dose-response relationship, which culminated at over 40 years of age with an OR of 13.13 (95\% CI: 4.63-37.26). The effect of increased maternal age was found to be monotonic and, by virtue of the logistic regression analysis, was independent of the paternal age's effect. Interestingly, the OR of ASD risk in this cohort increased dramatically with advancing maternal age category $(\mathrm{OR}=3.39,6.12,7.86$ and 13.13 for age categories $25-29,30-34,35-39$ and $\geq 40$ years, respectively) in a similar fashion than in studies done in other populations. ${ }^{25,26}$

There was no data to further test hypotheses on mechanisms that could underlie the maternal age findings. However, based on published literature, it is hypothesised that advancing maternal age is associated with an indirect secular effect of increased obstetric and neonatal complications (e.g. pre-pregnancy obesity, excessive weight gain during pregnancy, gestational diabetes, caesarean section and preterm deliveries); despite no causal linkage being found for this hypothesis, advanced maternal age's contribution to ASD risk is plausible due to documented risk of maternal and neonatal complications. ${ }^{7,27}$ Although this dataset lacked information about infertility, in vitro fertilisation treatment and intracytoplasmic sperm injection (ICSI), it has been postulated that offspring of mothers who underwent ICSI for conception (who also tend to be older) may have a statistically higher risk of having a child with autism. ${ }^{28}$ However, despite the multiple hypotheses postulated to explain the association between advanced maternal age and ASD, no clear conclusion has yet been reached in the literature.

The effect of advanced paternal age was also found to be significant in this study, although of lesser magnitude when compared to the robust association of maternal age and ASD risk. The OR distribution was lower than that for maternal age from 25 to 44 years $(\mathrm{OR}=2.20,2.36$ and 3.12 for age categories $30-$ 34, 35-39 and 40-44 years, respectively); however, the risk increase was statistically noteworthy and added to the effect of maternal age. The de novo mutations theory, which is linked to advanced paternal age, could be a plausible explanation for the effect. For example, among ASD cases from the Simons Simplex Collection, coding de novo mutations, including cases with copy number variants, contributed to approximately $30 \%$ of simplex cases and to $45 \%$ of female ASD cases. ${ }^{29}$ Surprisingly, there was a decrease in the effect among fathers $\geq 45$ years in the current study, which could be due to a selection bias or to the small sample size of older fathers. However, other possibilities related to special demographic characteristics and to the local context should be considered. For instance, the population in Oman is considered younger than Western communities with consanguinity marriage rates of approximately 52\%, which is much higher than in Western populations. ${ }^{19,20}$ The risk of ASD at advanced paternal age was explained by de novo mutations; however, that relationship might be different in a highly consanguineous population with increased prevalence of recessive alleles..$^{29,30}$ Therefore, the findings of the drop in paternal age's effect can support the multifactorial pathway theory with a different interplay of physio-pathological mechanisms in a diverse population context. ${ }^{18}$

Considering the importance of maternal and child health, this study's findings can assist in counselling with regards to the risks of pregnancy at an older age. The strength of this study stems from the large sample size and that it is the first study to assess the effect of advancing parental age in ASD risk in an Omani population. However, the data should be interpreted 
with caution, taking into consideration that ASD cases originated from a single centre along with a short study duration. While the control group electronic records were reviewed to ensure the absence of neurodevelopmental symptoms that could be related to ASD, the absence of direct clinical assessment of this control group could have led to misclassification, although this risk remains small. Moreover, if such misclassification occurred, it would have biased the results towards the null hypothesis and resulted in some underestimation of the associations reported on parental age and risk of autism. Furthermore, there is a possibility of unmeasured confounding variables and residual confounding, which may bias the risk associations found. Nevertheless, the importance of this initial observation arises from the similarities in demographic features in Gulf Corporation Council (GCC) countries, which necessitates more studies to verify such findings in particular, as well as to more generally investigate autism epidemiology, comorbidities, outcome and efficacy of treatment strategies.

\section{Conclusion}

Increased parental age was associated with a heightened risk of ASD in their offspring; the risk increase was more robust for advanced maternal age. Investigations of changes in maternal health with increasing age for several medical conditions (obesity, diabetes, etc.) that have been associated with an increased risk of ASD in offspring are needed. The OR distribution for paternal age was lower than that for maternal age from 25 to 44 years; however, the effect of paternal age was still statistically significant. Surprisingly, this study found a decrease in the OR with a paternal age of $\geq 45$ years, which requires further testing. This finding may support the multifactorial pathway hypothesis to explain the effect of parental age in ASD risk. Further studies are needed and, keeping in mind the similarities in demographic features among GCC populations, multicentre studies with larger sample sizes should be facilitated.

\section{CONFLICT OF INTEREST}

The authors declare no conflicts of interest.

\section{FUNDING}

No funding was received for this study.

\section{AUTHORS CONTRIBUTION}

WM and ABI conceptualised the idea and drafted the manuscript. SJ, WM, AG and ABI did the clinical assessment. The data collection was done by AAA-Z and MJ. EF revised the initial version of the manuscript. The data management and data analysis were done by SM and EF. EF, WM and ABI revised the final manuscript. All authors approved the final version of the manuscript.

\section{References}

1. American Psychiatric Association. Diagnostic and Statistical Manual of Mental Disorders. 5th Ed. Washington: American Psychiatric Association, 2013.

2. Maenner MJ, Shaw KA, Baio J; EdS1, Washington A, Patrick M, et al. Prevalence of autism spectrum disorder among children aged 8 years - Autism and Developmental Disabilities Monitoring Network, 11 Sites, United States, 2016. MMWR Surveill Summ 2020; 69:1-12. https://doi.org/10.15585/MMWR.SS6904A1.

3. Elsabbagh M, Divan G, Koh Y, Kim YS, Kauchali S, Marcín C, et al. Global prevalence of autism and other pervasive developmental disorders. Autism Res 2012; 160-79. https://doi.org/1 0.1002/aur.239.

4. Al-Mamri W, Idris AB, Dakak S, Al-Shekaili M, Al-Harthi Z, Alnaamani AM, et al. Revisiting the prevalence of autism spectrum disorder among omani children a multicentre study. Sultan Qaboos Univ Med J 2019; 19:e305-9. https://doi.org/10.18295/ squmj.2019.19.04.005.

5. Polyak A, Kubina RM, Girirajan S. Comorbidity of intellectual disability confounds ascertainment of autism: implications for genetic diagnosis. Am J Med Genet Part B Neuropsychiatr Genet 2015; 168:600-8. https://doi.org/10.1002/ajmg.b.32338.

6. Hultman CM, Sparén P, Cnattingius S. Perinatal risk factors for infantile autism. Epidemiology 2002; 13:417-23. https://doi. org/10.1097/00001648-200207000-00009.

7. Getahun D, Fassett MJ, Peltier MR, Wing DA, Xiang AH, Chiu V, et al. Association of perinatal risk factors with autism spectrum disorder. Am J Perinatol 2017; 34:295-304. https:// doi.org/10.1055/s-0036-1597624.

8. Xiang AH, Wang X, Martinez MP, Walthall JC, Curry ES, Page K, et al. Association of maternal diabetes with autism in offspring. JAMA 2015; 313:1425-34. https://doi.org/10.1001/ jama.2015.2707.

9. Jo H, Schieve LA, Sharma AJ, Hinkle SN, Li R, Lind JN. Maternal prepregnancy body mass index and child psychosocial development at 6 years of age. Pediatrics 2015; 135:e1198-209. https://doi.org/10.1542/peds.2014-3058.

10. Wang C, Geng H, Liu W, Zhang G. Prenatal, perinatal, and postnatal factors associated with autism: A meta-analysis. Medicine (Baltimore) 2017; 96:e6696. https://doi.org/10.1097/ MD.0000000000006696.

11. Reichenberg A, Gross R, Weiser M, Bresnahan M, Silverman J, Harlap S, et al. Advancing paternal age and autism. Arch Gen Psychiatry 2006; 63:1026-32. https://doi.org/10.1001/archps yc.63.9.1026.

12. Durkin MS, Maenner MJ, Newschaffer CJ, Lee LC, Cunniff CM, Daniels JL, et al. Advanced parental age and the risk of autism spectrum disorder. Am J Epidemiol 2008; 168:1268-76. https:// doi.org/10.1093/aje/kwn250.

13. Parner ET, Baron-Cohen S, Lauritsen MB, Jørgensen $M$, Schieve LA, Yeargin-Allsopp M, et al. Parental age and autism spectrum disorders. Ann Epidemiol 2012; 22:143-50. https:// doi.org/10.1016/j.annepidem.2011.12.006.

14. Sandin S, Schendel D, Magnusson P, Hultman C, Surén P, Susser E, et al. Autism risk associated with parental age and with increasing difference in age between the parents. Mol Psychiatry 2016; 21:693-700. https://doi.org/10.1038/mp.2015.70. 
15. Wu S, Wu F, Ding Y, Hou J, Bi J, Zhang Z. Advanced parental age and autism risk in children: A systematic review and metaanalysis. Acta Psychiatr Scand 2017; 135:29-41. https://doi.org/1 $0.1111 /$ acps.12666.

16. O’Roak BJ, Vives L, Girirajan S, Karakoc E, Krumm N, Coe BP, et al. Sporadic autism exomes reveal a highly interconnected protein network of de novo mutations. Nature 2012; 485:246-50. https://doi.org/10.1038/nature10989.

17. O’Roak BJ, Vives L, Fu W, Egertson JD, Stanaway IB, Phelps IG, et al. Multiplex targeted sequencing identifies recurrently mutated genes in autism spectrum disorders. Science 2012; 338:1619-22. https://doi.org/10.1126/science.1227764.

18. Janecka M, MillJ, BassonMA, GorielyA, Spiers H, ReichenbergA, et al. Advanced paternal age effects in neurodevelopmental disorders-review of potential underlying mechanisms. Transl Psychiatry 2017; 7:e1019. https://doi.org/10.1038/tp.2016.294.

19. World Health Organization. Global Health Observatory data repository. From: https://apps.who.int/gho/data/view.main. POP2040 Accessed: Jul 2020

20. Islam MM. The practice of consanguineous marriage in Oman: Prevalence, trends and determinants. J Biosoc Sci 2012; 44:571-94. https://doi.org/10.1017/S0021932012000016.

21. Al Farsi M, West DJ Jr. Use of electronic medical records in Oman and physician satisfaction. J Med Syst 2006; 30:17-22. https://doi.org/10.1007/s10916-006-7399-7.

22. Lyall K, Croen L, Daniels J, Fallin MD, Ladd-Acosta C, Lee BK et al. The changing epidemiology of autism spectrum disorders. Annu Rev Public Health 2017; 38 :81-102. https://doi.org/10.11 46/annurev-publhealth-031816-044318.

23. National Centre for Statistics and Information. Statistical Year Book: 2017. From: https://www.ncsi.gov.om/Elibrary/Li braryContentDoc/bar Statistical\%20Year\%20Book\%202017 c2111831-e13a-4075-bf7b-c4b5516e1028.pdf Accessed: Oct 2020.
24. Fountain C, King MD, Bearman PS. Age of diagnosis for autism: Individual and community factors across 10 birth cohorts. J Epidemiol Community Health 2011; 65:503-10. https://doi. org/10.1136/jech.2009.104588.

25. Croen L, Najjar D, Fireman B, Grether JK. Maternal and paternal age and risk of autism spectrum disorders. Arch Pediatr Adolesc Med 2007; 161:334-40. https://doi.org/10.1001/ archpedi.161.4.334.

26. Shelton JF, Tancredi DJ, Hertz-Picciotto I. Independent and dependent contributions of advanced maternal and paternal ages to autism risk. Autism Res 2010; 3:30-9. https://doi. $\operatorname{org} / 10.1002 /$ aur.116.

27. Jacobsson B, Ladfors L, Milsom I. Advanced maternal age and adverse perinatal outcome. Obstet Gynecol 2004; 104:727-33. https://doi.org/10.1097/01.AOG.0000140682.63746.be.

28. Sandin S, Nygren KG, Iliadou A, Hultman CM, Reichenberg A. Autism and mental retardation among offspring born after in vitro fertilization. JAMA 2013; 310:75-84. https://doi. org/10.1001/jama.2013.7222.

29. Iossifov I, O'Roak BJ, Sanders SJ, Ronemus M, Krumm N, Levy D, et al. The contribution of de novo coding mutations to autism spectrum disorder. Nature 2014; 515:216-21. https:// doi.org/10.1038/nature13908.

30. Al-Thihli K, Al-Murshedi F, Al-Hashmi N, Al-Mamari W, Islam MM, Al-Yahyaee SA. Consanguinity, endogamy and inborn errors of metabolism in Oman: A cross-sectional study. Hum Hered 2014; 77:183-8. https://doi.org/10.1159/000362686. 